\title{
Proposta de um modelo híbrido multidimensional para apoiar o processo de avaliação de estratégia tecnológica
}

\author{
Sheila Reinehr ${ }^{1}$, Marcelo Pessôa ${ }^{2}$, Fernando Laurindo ${ }^{2}$, Robert Burnett ${ }^{1}$ \\ ${ }^{1}$ Pontifícia Universidade Católica do Paraná \\ Rua Imaculada Conceição, 1155 - Curitiba - PR - Brasil \\ ${ }^{2}$ Escola Politécnica - Universidade de São Paulo [USP] \\ Av. Professor Almeida Prado, travessa 2, n. 128 - São Paulo - SP - Brasil \\ \{sheila.reinehr, robert.burnett\}@pucpr.br, \{mpessoa, fkblau\}@usp.br
}

\begin{abstract}
Resumo. Avaliar a melhor estratégia tecnológica na aquisição de produtos e serviços de TI não é uma atividade trivial e, em geral, exige uma perspectiva multidimensional. Existem diferentes métodos de avaliação aplicáveis aos diferentes tipos software, dependendo da abrangência e dos efeitos que a aplicação pode ter sobre os negócios da organização. Este artigo propõe um modelo híbrido multidimensional de avaliação que leva em consideração quatro dimensões de preocupação: Negócios, Arquitetura, Processo e Organização [NAPO, do original BAPO]. Neste artigo, o contexto de uma empresa do ramo de cooperativas médicas é analisado.
\end{abstract}

Abstract. Evaluating the best technology strategy in IT products and services acquisition is not an ordinary task. It often requires a multidimensional perspective. There are different evaluation methods applicable to different kinds of products, depending on the focus and the effects that such application has on the business. This paper proposes a hybrid multidimensional evaluation method that takes into account four concern dimensions: Business, Architecture, Process and Organization [BAPO]. In this paper an organization of the medical cooperative segment is analyzed.

\section{Introdução}

A Tecnologia da Informação [TI] é uma área relativamente nova se comparada a outras áreas do conhecimento humano e por esse motivo encontra-se em plena evolução. Durante as últimas décadas o que se viu foi a invenção de novos equipamentos e softwares, viabilizando a criação de produtos e serviços cada vez mais sofisticados. Em [BOSCH, 2000] o autor delineia esta situação da seguinte forma: "Software está se tornando mais e mais uma parte integrante da sociedade e, se, por alguma razão, algum dia todo o software parasse de funcionar, o efeito no mundo ocidental seria catastrófico. Embora as pessoas que trabalham em disciplinas técnicas estejam cientes deste fato há muitos anos, não era claro para o público em geral, até que o problema do ano 2000 e suas conseqüências tivessem sido discutidos abertamente na mídia” (p. 1).

O termo tecnologia da informação firmou-se a partir da década de 80, substituindo expressões como informática e processamento de dados, anteriormente de uso disseminado. Em [LAURINDO, 2002], o autor utiliza o termo incluindo: “... o uso de hardware, software, telecomunicações, automação, recursos multimídia e demais 
recursos envolvidos, sem deixar de considerar os sistemas de informação, serviços, negócios, usuários e as relações complexas envolvidas”. Desde os idos tempos do termo processamento de dados, até os dias atuais, muita coisa no mundo da TI mudou, além de simplesmente a utilização de um termo novo. A complexidade e o tamanho dos projetos de TI, especialmente aqueles envolvendo software, quer como componentes isolados ou como parte integrante de um sistema, têm crescido de tal forma que se torna cada vez mais difícil assegurar seu sucesso. Essa incerteza tem levado as empresas a analisar com mais critério e rigor, seus investimentos em TI. Apesar da pressão exercida pelo mercado continuar levando à busca de inovação constante, melhor qualidade e menor custo; os investimentos em TI têm sido feitos de forma mais seletiva e cautelosa, com grande ênfase para os aspectos de planejamento e retorno sobre o investimento.

Como a TI passou a permear todos os setores da economia, os executivos passaram a decidir por investimentos [na sua maioria de grande valor] em projetos sobre os quais não tinham plena convicção de sucesso. No caso de setores que não atuam diretamente em TI, muitas vezes os administradores são profissionais de engenharia, saúde, comunicação social etc. Esses profissionais, de uma forma geral, não se sentem seguros para tomar decisões quando palavras relacionadas às tecnologias emergentes, como business intelligence, voice over IP, knowledge management entre outras, passam a fazer parte da pauta de suas reuniões.

Paralelamente a isto, a área de TI passou a buscar, de forma incessante, a famosa "bala de prata" [BROOKS, 1995] para conseguir aumentar a sua produtividade na mesma proporção que crescia a potência dos processadores. Nesta esteira, novos paradigmas de desenvolvimento [orientação a objetos, desenvolvimento orientado a reuso, engenharia de famílias de sistemas etc.], metodologias [processo unificado, metodologias ágeis etc.], modelos de qualidade [como CMMI, ITIL, COBIT etc.] e normas internacionais [ISO/IEC 12207, ISO/IEC 9126 etc.] foram sendo criados e se expandiram, gerando uma miríade de opções para solucionar a crise de software.

Com tantas dúvidas de um lado [o adquirente] e com tantas opções do outro [os fornecedores], como se decidir pela melhor alternativa no momento de definir uma estratégia tecnológica? Como fazer isto de forma ágil e com baixo custo, sem que tenha que se mobilizar uma grande quantidade de especialistas? A questão a que este artigo se propõe a analisar é: "Que modelo pode apoiar uma empresa adquirente de produtos de TI a selecionar a melhor alternativa tecnológica de forma ágil, utilizando como base os modelos de qualidade líderes de mercado?”.

A seção 2 deste artigo descreve a metodologia de pesquisa adotada; a seção 3, o cenário da pesquisa; a seção 4, a ação proposta, ou seja, o modelo híbrido multidimensional desenvolvido; a seção 5, a implementação da ação, ou seja, o uso do modelo; a seção 6 discute os resultados obtidos; a seção 7 apresenta conclusões e trabalhos futuros, seguida das referências bibliográficas.

\section{Metodologia de Pesquisa}

Devido às suas características, a pesquisa realizada se enquadra como sendo do tipo Pesquisa-Ação [COUGHLAN; COGHLAN, 2002], uma vez que possui: 
- foco na pesquisa em ação: os pesquisadores atuam para resolver problemas organizacionais juntamente com aqueles que vivem o problema diretamente;

- pesquisa participativa: o pesquisador é, em geral, um agente externo ao ambiente e atua como facilitador e apoio nas reflexões sobre a pesquisa; os membros internos ao ambiente atuam diretamente na avaliação dos resultados;

- pesquisa concorrente à ação: objetiva-se tornar a ação mais efetiva à medida em que conhecimentos científicos são agregados;

- seqüência de eventos [ciclos iterativos de obtenção de dados] e uma abordagem para a solução de problemas [abordagem de análise de fatos e experimentação].

Segundo os autores, um projeto de Pesquisa-Ação utiliza um processo emergente, cuja forma se delineia à medida em que desenrola a ação e que dela depende diretamente para adquirir forma. Ainda, em [COUGHLAN; COGHLAN, 2002], os autores sugerem uma abstração genérica, utilizando uma abordagem iterativa e incremental, conforme ilustrado na Figura 1.

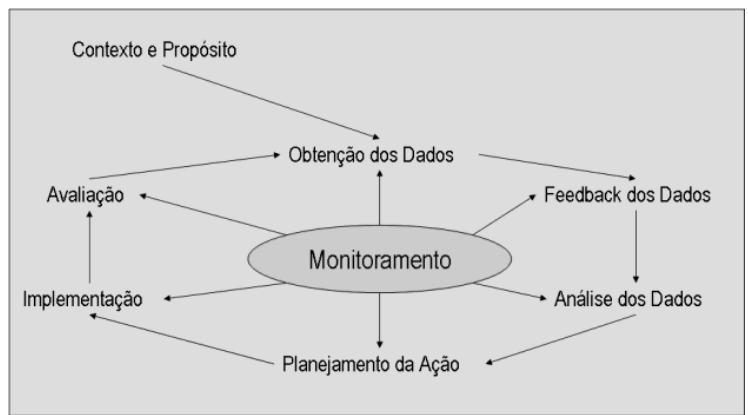

Figura 1. Fases da Pesquisa-Ação, adaptado de [COUGHLAN; COGHLAN, 2002].

A etapa de Contexto e Propósito pode ser considerada como uma etapa preliminar, na qual se compreende o contexto de inserção da pesquisa e se delineia o domínio e o problema a ser resolvido. As demais, podem se desenrolar cíclica e iterativamente, até que os resultados obtidos possam ser considerados satisfatórios, sendo combinadas de forma determinada pelo próprio desenrolar da ação. No centro do processo destaca-se a atividade de Monitoramento, essencial para o processo de reflexão necessário à adequação da ação aos propósitos da pesquisa e para a própria reformulação do processo aplicado. A pesquisa relatada neste artigo baseia-se no proposto por [COUGHLAN; COGHLAN, 2002] e foi desenvolvida utilizando as etapas sumarizadas a seguir e detalhadamente descritas nas próximas seções:

Etapa 0 - Contexto e propósito: em se tratando de uma Pesquisa-Ação, ou, ainda, uma pesquisa em ação, a etapa preliminar visou identificar o contexto de inserção do problema e o propósito da pesquisa: avaliação de estratégia tecnológica aplicada ao domínio de cooperativas de saúde. A compreensão do domínio e o entendimento dos instrumentos disponíveis no referencial bibliográfico constituíram a base para o planejamento da ação.

Etapa 1 - Obtenção dos dados: o primeiro passo da pesquisa foi a coleta inicial de dados de campo, especialmente relacionados ao segmento de mercado, projeções, crescimento dos participantes etc. Conforme descrito em [COUGHLAN; COGHLAN, 2002], nesta 
fase pode-se obter dados que são considerados hard [obtidos diretamente da observação do desenrolar das atividades da unidade organizacional ou de relatórios de desempenho] e $s o f t$ [obtidos pelas análises, entrevistas e outras fontes]. Foram ainda coletados dados do referencial teórico disponível referente aos modelos e normas que apóiam a avaliação para aquisição.

Etapa 2 - Análise dos dados: em seguida foram analisados os dados sob a ótica dos modelos existentes no mercado e sua aplicabilidade à situação específica do contexto, permitindo projetar as etapas que se seguiriam.

Etapa 3 - Planejamento da ação: esta fase da pesquisa foi traduzida na prática pela construção de um modelo conceitual inicial proposto para ser aplicado como referência para a comparação entre as possíveis soluções de estratégia tecnológica. Este modelo, construído inicialmente com base no referencial teórico coletado na etapa 1 e analisado na etapa 2, acrescido de dados obtidos nas entrevistas com os membros internos da organização alvo; foi posteriormente refinado, como se verá.

Etapa 4 - Implementação da ação: esta etapa foi implementada na prática pela aplicação do modelo conceitual proposto (ação), sobre os fornecedores apontados pelo adquirente como sendo potenciais para a migração da estratégia.

Etapa 5 - Avaliação dos resultados da aplicação da ação: a avaliação dos resultados se deu em duas etapas. A primeira, imediatamente após a execução da ação (uso do modelo de avaliação na situação contexto). Outra, após um período de utilização da estratégia definida, que visou comprovar a eficácia da avaliação sobre o contexto estudado.

\section{Contexto e propósito: descrição do cenário da pesquisa}

A etapa preliminar, Contexto e Propósito, seguida da etapa 1 (obtenção dos dados), levou à compreensão do cenário da pesquisa: o setor de cooperativas médicas. $\mathrm{O}$ setor de saúde suplementar no Brasil reúne mais de 2000 empresas operadoras. Essa rede prestadora de serviços, representada por profissionais de saúde, hospitais, laboratórios e clínicas, atende a mais de 34 milhões de consumidores que utilizam a assistência à saúde para realizar consultas, exames ou internações.

A Agência Nacional de Saúde Suplementar - ANS, criada em janeiro/ 2000 e vinculada ao Ministério da Saúde, é o órgão responsável pela regulamentação. A ANS classifica as empresas que trabalham com saúde complementar em: seguradoras especializadas em saúde, administradoras, cooperativas médicas, cooperativas odontológicas, instituições filantrópicas, autogestões (patrocinadas ou não), medicina de grupo e odontologia de grupo. Todas as empresas que operam com planos de saúde complementar, independentemente do seu tipo, são regidas pela Lei 9656/98 e pela Medida Provisória [MP] 2177-44, e são fiscalizadas pela ANS [ANS, 2009].

Algumas das ações da ANS para o controle efetivo do setor incluem o acompanhamento e a análise das informações detalhadas prestadas pelas operadoras sobre seu desempenho financeiro. Essas informações, além de outras relativas ao setor, são disponibilizadas para toda a comunidade e são também utilizadas para controlar sua operação, protegendo os direitos do consumidor. A ANS representa para as operadoras de plano de saúde papel similar ao do Banco Central (BACEN) e ao da Comissão de Valores Mobiliários (CVM), para o setor financeiro. 
De forma a manter-se atuando e competindo no mercado, a empresa de saúde complementar precisa utilizar, de forma intensa e eficiente, os recursos de TI. Esse é um instrumento importante para que ela consiga, não somente agilizar o atendimento a seus clientes, mas cumprir as regulamentações e exigências do órgão fiscalizador, a ANS.

Há alguns anos atrás, a empresa analisada optou por não desenvolver seus próprios sistemas de informação, mas utilizar o sistema de outra entidade do mesmo grupo, pagando, para isso, uma taxa mensal de utilização e manutenção. O sistema contratado auxilia a cooperativa a operar os seus planos de saúde, oferecendo recursos operacionais (cadastro, faturamento, liberação, repasses), relatórios gerenciais e repasses para a ANS, o órgão fiscalizador. Como este é um setor bastante fiscalizado e regulamentado, a exemplo dos bancos, constantes adequações legais são necessárias, estando o sistema em permanente estado de atualização. No entanto, a empresa fornecedora está descontinuando a versão atual e está oferecendo a opção de migração para uma nova versão, contendo as mesmas funcionalidades, porém tecnologicamente mais avançada e com custos de operação potencialmente menores. Além disso, esta nova versão abre novas possibilidades, como: integração inter-organizacional com os prestadores de serviços e implantação de funcionalidades de business intelligence.

A empresa encontrava-se diante de um importante momento de decisão quanto à sua estratégia de TI. Poderia manter sua decisão de terceirização, tomada anos atrás, ou poderia optar por iniciar o seu próprio desenvolvimento de software. Caso optasse por continuar com o processo de terceirização, teria, ainda três opções: continuar utilizando o sistema atual (que não seria mais evoluído e nem adaptado às exigências legais); migrar para uma nova solução oferecida pelo mesmo fornecedor ou migrar para uma solução oferecida por outra empresa do mesmo grupo de cooperativas médicas. Essa última alternativa foi o motivador principal da necessidade de avaliar de forma sistemática as alternativas. Apesar de ser apontada como a solução do futuro e de estar em uma curva crescente de adoção, havia indícios de que o fornecedor não conseguiria suportar um grande número de clientes.

Como uma empresa do tipo cooperativa, seu maior objetivo, além de proporcionar um bom atendimento aos usuários finais (clientes do plano de saúde), é oferecer uma forma cooperada e vantajosa de compartilhar despesas entre os conveniados (profissionais de saúde, laboratórios, hospitais e clínicas), não deixando de cumprir todas as exigências legais do setor.

\section{Planejamento da ação: modelo de avaliação proposto}

Para chegar ao planejamento da ação e, consequentemente, à execução da ação em si, identificou-se a necessidade de execução de 3 passos intermediários, representados por mais uma iteração da etapa 1 (obtenção de dados) e etapa 2 (análise de dados), desta vez com o objetivo de levantar as informações sobre os modelos disponíveis no referencial teórico e que poderiam ser utilizados no processo de planejamento da ação. Na prática, isto se refletiu na execução das seguintes atividades:

- Identificação das bases conceituais para o modelo proposto

- Identificação dos atributos e conceitos para a avaliação

- Definição do instrumento de avaliação

- Análise crítica do modelo em relação ao contexto de aplicação 


\subsection{Identificação das bases conceituais para o modelo proposto}

Segundo [FARBEY et al., 1995], é necessário que as empresas empreguem métodos diferentes e apropriados para avaliar cada tipo de aplicação. Nenhum método é genérico o suficiente para ser aplicado a qualquer tipo de organização, qualquer tipo de sistema de informação e qualquer objetivo de avaliação. Uma análise crítica comparativa entre os modelos de avaliação, tais como os modelos propostos por [NOLAN, 1973] e [McFARLAN, 1984], pode ser encontrada em [FARBEY et al., 1995] e também em [LAURINDO, 2002].

A Figura 2 ilustra os oito degraus propostos pelos autores em [FARBEY et al.,1995] em sua escada de benefícios. Cada degrau representa um tipo de mudança e, conseqüentemente, um tipo de aplicação. Degraus mais altos indicam benefícios potenciais maiores, porém, incertezas também maiores na avaliação. Para cada tipo específico de aplicação, determinados tipos de avaliação são mais adequados. Para os degraus inferiores, pode-se usar métodos mais determinísticos, enquanto que nos degraus superiores, as avaliações se tornam mais subjetivas e complexas.

\begin{tabular}{|c|c|c|}
\hline & TIPO DE APLICAÇÃO & TIPO DE AVALIAÇÃO \\
\hline 8 & $\begin{array}{l}\text { Transformação do negócio: mudanças mais } \\
\text { complexas onde Tl é apenas um dos componentes }\end{array}$ & $\begin{array}{l}\text { Avaliação do pacote de mudanças como um todo. } \\
\text { Escolha da tecnologia que facilita a maior } \\
\text { quantidade de mudanças. }\end{array}$ \\
\hline 7 & Sistemas Estratégicos & $\begin{array}{l}\text { Avaliações baseadas na compreensão da situação } \\
\text { do negócio (especialmente a situação de } \\
\text { competição) e uma cuidadosa análise de riscos. }\end{array}$ \\
\hline 6 & Sistemas inter-organizacionais & $\begin{array}{l}\text { Avaliar o custo } \mathrm{x} \text { beneficio da perda de } \\
\text { flexibilidade em relação aos ganhos do sistema } \\
\text { interligado. }\end{array}$ \\
\hline 5 & $\begin{array}{l}\text { Infra-estrutura: sistemas de automação de } \\
\text { escritórios, telecomunicações etc. }\end{array}$ & $\begin{array}{l}\text { Demonstrar a ligação entre o investimento em } \\
\text { infra-estrutura e os beneficios dos projetos que } \\
\text { serão desenvolvidos sobre ela. }\end{array}$ \\
\hline 4 & $\begin{array}{l}\text { Sistemas de Informações Gerenciais (SIG) e } \\
\text { Sistemas de Apoio à Decisão (SAD) }\end{array}$ & Valor agregado potencial. Melhor e pior caso \\
\hline 3 & $\begin{array}{l}\text { Sistemas de Valor Agregado Direto: além de } \\
\text { automatizar, adicionam valor }\end{array}$ & $\begin{array}{l}\text { Técnicas experimentais (como testes de campo, } \\
\text { prototipação). }\end{array}$ \\
\hline 2 & $\begin{array}{l}\text { Automação: substituir métodos existentes para } \\
\text { reduzir custos. }\end{array}$ & $\begin{array}{l}\text { Medição direta do trabalho (produtividade). } \\
\text { Métodos de pesquisa operacional (modelagem e } \\
\text { simulações). }\end{array}$ \\
\hline 1 & $\begin{array}{l}\text { Mudanças obrigatórias: leis, mudanças para } \\
\text { sobrevivência do negócio etc. }\end{array}$ & $\begin{array}{l}\text { Análise técnica clássica de custo } \mathrm{x} \text { beneficio entre } \\
\text { as alternativas tecnológicas. }\end{array}$ \\
\hline
\end{tabular}

Figura 2. Escada de Benefícios, adaptada de [Farbey et al. ,1995].

Segundo os mesmos autores, no mundo real existem poucos casos em que se consegue classificar uma determinada mudança de forma absoluta em um dos degraus. Nesse caso, é recomendado que se escolha os degraus mais altos, pois é melhor, em uma avaliação, superestimar os riscos do que subestimá-los. Como o modelo pretendido visa atender a uma gama genérica de situações, além do próprio objeto da pesquisa-ação, optou-se por considerá-lo como situado no último degrau, no qual as mudanças ocasionadas pelo produto são mais abrangentes e diretamente ligadas à competitividade.

Para estruturar o modelo conceitual optou-se pela abordagem BAPO [Business, Architecture, Process and Organization], neste trabalho traduzida para NAPO [Negócios, Arquitetura, Processo e Organização], conforme ilustra a Figura 3, adaptada de [WIJNSTRA, 2002]. A dimensão de Negócios avalia a forma de obter lucros da organização, neste trabalho interpretado como sendo os aspectos relacionados às 
questões financeiras da aquisição. A dimensão Organização neste trabalho reflete a estrutura da organização fornecedora para prover o produto e/ou serviços a ele associados. A dimensão Arquitetura está sendo refletida aqui como os aspectos relacionados aos requisitos da aplicação em si [produto], quer sejam funcionais ou não funcionais. E, finalmente, a dimensão Processo aqui será refletida como sendo as práticas empregadas no processo de desenvolvimento e manutenção que o fornecedor utiliza.

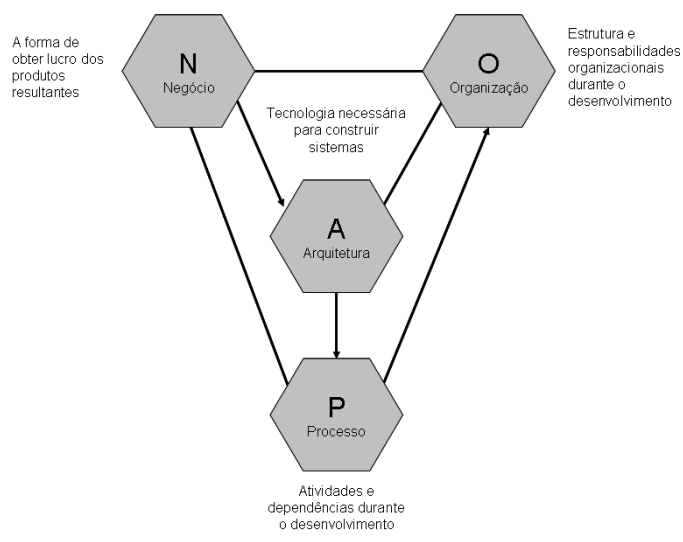

Figura 3. Estrutura de critérios, adaptada de [WIJNSTRA, 2002].

Para atender a cada uma das dimensões do NAPO optou-se, quando possível, pelo uso de modelos consagrados internacionalmente e de grande aceitação no mercado, de modo a permitir sua generalização e disseminação. Desta forma:

- A [Arquitetura]: para definir os atributos referentes à aplicação em si, optou-se pelo uso da norma ISO/IEC 9126 - Avaliação de produto de software Características de qualidade e diretrizes para o seu uso [ABNT, 1996].

- P [Processo]: para os atributos referentes ao processo utilizado pelo fornecedor, optou-se pelo uso do modelo internacional CMMI - Capability Maturity Model Integration for Development [SEI, 2006], inicialmente com foco nas áreas de processo de nível 2 e algumas de nível 3, no entanto adaptadas.

- $\mathrm{N}$ [Negócio]: para os atributos referentes ao negócio optou-se por uma forma empírica, utilizando inicialmente a experiência profissional e acadêmica dos autores e, posteriormente, um refinamento através de entrevistas com o corpo gerencial da empresa onde foi realizada a pesquisa.

- O [Organização]: para os atributos organizacionais a mesma estratégia da dimensão de Negócios foi adotada.

\subsection{Definição dos atributos e conceitos para avaliação}

A partir dos modelos de referência selecionados, foram definidos os atributos que poderiam melhor contribuir para a realização de uma avaliação sob a ótica do adquirente, ou seja, sem necessariamente possuir um conceito aprofundado da área de tecnologia da informação, respeitando a premissa de agilidade estabelecida.

A Tabela 1 apresenta os critérios sugeridos para a avaliação do processo que o fornecedor utiliza no dia a dia para manter o produto que o adquirente está analisando. Estes critérios são o resultado da definição inicial empírica dos pesquisadores, do uso da 
versão 1.1 do modelo CMMI e do refinamento ocorrido após a liberação da versão 1.2 do modelo CMMI [CMMI for Development] descrita em [SEI, 2006]. Se uma avaliação mais detalhada da dimensão Processo for requerida, recomenda-se uma avaliação formal, baseada no modelo completo CMMI ou similar, como o modelo nacional MPSBR [SOFTEX, 2006].

Tabela 1. Critérios para avaliação da dimensão $P$ (Processo).

\begin{tabular}{|l|}
\hline \multicolumn{1}{|c|}{ CRITÉRIOS PARA AVALIAÇÃO DA DIMENSÃO P (PROCESSO) } \\
\hline PROCESSO GERENCIADO \\
- Planejamento do Projeto: O fornecedor possui um processo de planejamento que garante que o cronograma é gerado \\
com base em estimativas documentadas, prevendo pontos de checagem intermediários. As estimativas se baseiam em \\
dados históricos. \\
- Monitoramento e Controle do Projeto: O fornecedor acompanha o desempenho dos projetos de software de acordo \\
- - Gem os pontos de checagem planejados e re-planeja, se necessário. \\
gerência de Configuração: O fornecedor utiliza o conceito de versões do produto, possuindo um processo de \\
qualquer momento, recuperorar uma versão anterior. \\
- Gerência de Requisitos: O fornecedor possui um processo definido para o registro dos requisitos, priorização e \\
alocação desses requisitos em versões do software, bem como registros de todas as alterações nos requisitos. \\
- Garantia da Qualidade: O fornecedor utiliza um processo de garantia de qualidade efetuado por pessoas externas à \\
equipe do projeto. Todos os problemas encontrados são acompanhados até o seu fechamento. \\
Métricas: O fornecedor possui um conjunto de métricas que apóia efetivamente seus processos de gestão na área de TI. \\
PROCESSO DEFINIDO \\
- Engenharia de Software: O fornecedor possui uma metodologia de desenvolvimento de sistemas que estabelece o \\
modelo de ciclo de vida a ser usado, os métodos, as ferramentas e os padrões a serem seguidos. Todos os projetos são \\
desenvolvidos seguindo essa metodologia. Todas as etapas do ciclo de vida de desenvolvimento são tratadas: \\
desenvolvimento de requisitos, elaboração da solução técnica, testes (em diversos níveis), integração, homologação e \\
implantação. \\
- Treinamento: O fornecedor possui um programa de treinamento e capacitação constante para os profissionais que \\
compõem a equipe de desenvolvimento, bem como para novos integrantes, quando for o caso. \\
Melhorias: Existem iniciativas gerenciadas de melhoria das práticas de desenvolvimento e gestão da área de TI.
\end{tabular}

Caso a organização já possua uma certificação de qualidade, o mais importante é investigar o quanto as práticas que foram certificadas efetivamente são aplicadas ao desenvolvimento e manutenção do produto que está sendo analisado. Esta é uma preocupação muito pertinente, uma vez que as avaliações utilizam como amostra um certo número de projetos e não necessariamente o projeto que gerou o produto estava presente na amostra ou utilizou as práticas avaliadas. Outro cuidado pertinente em relação às empresas que possuem algum nível de certificação é identificar especificamente o escopo da certificação, aquilo que o modelo CMMI chama de organização [SEI, 2006]. Como uma empresa pode avaliar apenas uma parte de sua equipe de desenvolvimento, pode ser que a unidade que desenvolveu o projeto que gerou o produto não seja a que foi oficialmente avaliada.

A Tabela 2 mostra a proposta dos atributos que foram inicialmente definidos para serem utilizados na avaliação do produto de software. Apesar do nome desta dimensão ser Arquitetura, os atributos selecionados dizem respeito à aplicação como um todo, ou seja, seus aspectos funcionais e não-funcionais, muitos deles, certamente, influenciados pela qualidade da arquitetura. No caso do contexto analisado, não fazia parte do escopo da pesquisa uma análise arquitetural detalhada das soluções.

Caso seja necessário, em outro tipo de contexto, focar mais detalhadamente os aspectos de arquitetura em si, recomenda-se a adoção de um modelo mais específico para esta finalidade, como o método ATAM (Architecture Tradeoff Analysis Method) 
proposto por pesquisadores do SEI - Software Engineering Institute e descrito em detalhes em [CLEMENTS et al, 2001]. O método apóia a análise do impacto das decisões arquiteturais sobre os atributos de qualidade do produto. Por ser uma análise mais técnica, ela não poderia ser conduzida por pessoas inexperientes em desenvolvimento de software e, certamente iria requerer a participação, do lado do adquirente, de um arquiteto de software especializado.

Tabela 2. Critérios para avaliação da dimensão A (Arquitetura).

\begin{tabular}{|c|c|}
\hline \multicolumn{2}{|r|}{ CRITÉRIOS PARA AVALIAÇÃO DA DIMENSÃO A (ARQUITETURA) } \\
\hline & $\begin{array}{l}\text { IONALIDADE } \\
\text { Adequação: As funções do sistema são adequadas às tarefas especificadas. } \\
\text { Acurácia: O sistema gera resultados precisos e corretos. } \\
\text { Interoperabilidade: O sistema interage adequadamente com outros sistemas. } \\
\text { Conformidade: O sistema está em conformidade com as normas e diretrizes da ANS } \\
\text { Segurança de Acesso: O sistema possui mecanismos que controlam o acesso aos dados e programas. }\end{array}$ \\
\hline & $\begin{array}{l}\text { IABILIDADE } \\
\text { Maturidade: O sistema consegue tratar de forma adequada os defeitos de modo que não se tornem falhas. } \\
\text { Tolerância a Falhas: O sistema mantém nível especificado de desempenho em caso de falha. } \\
\text { Recuperabilidade: O sistema possui capacidade de restaurar seu nível de desempenho e recuperar os dados } \\
\text { diretamente afetados, em caso de falha. } \\
\text { Contingência: O fornecedor possui procedimentos de contingência para o caso de falhas do software e do } \\
\text { hardware, quando se aplicar. }\end{array}$ \\
\hline & $\begin{array}{l}\text { ILIDADE } \\
\text { Inteligibilidade: O sistema apresenta facilidade no entendimento do seu uso. } \\
\text { Apreensibilidade: O sistema é fácil de aprender a usar. } \\
\text { Operacionalidade: O sistema é fácil de ser operado e controlado. }\end{array}$ \\
\hline & $\begin{array}{l}\text { ÊNCIA } \\
\text { Comportamento em Relação ao Tempo: O sistema tem tempo de resposta de acordo com o especificado. } \\
\text { Comportamento em Relação a Recursos: O sistema utiliza os recursos de hardware e software básico de } \\
\text { forma racional e de acordo com níveis especificados. }\end{array}$ \\
\hline & $\begin{array}{l}\text { JTENIBILIDADE } \\
\text { Analisabilidade: O sistema possui facilidade para diagnosticar causas de falhas. } \\
\text { Modificabilidade: O sistema é facilmente modificável em casos de defeitos e novas implementações. } \\
\text { Estabilidade: O sistema evita efeitos inesperados após modificações. } \\
\text { Testabilidade: O sistema é facilmente validado após modificações. }\end{array}$ \\
\hline & $\begin{array}{l}\text { ABILIDADE } \\
\text { Adaptabilidade: O sistema é facilmente adaptado para outros ambientes. } \\
\text { Capacidade para ser instalado: O sistema é facilmente instalado em ambiente especificado. } \\
\text { Conformidade: O sistema utiliza padrões ou convenções que o tornam portável. } \\
\text { Capacidade para substituir: O sistema possui facilidades para substituir outro sistema. }\end{array}$ \\
\hline
\end{tabular}

A Tabela 3 mostra os critérios que foram definidos para a dimensão de Negócios do modelo. Assim como nas demais dimensões, diversos são os critérios que podem ser utilizados para avaliar o aspecto Negócio, no entanto, como uma das premissas era prover um modelo para avaliação ágil e simplificada, optou-se por uma análise dos custos comparativos entre as soluções. Caso mais detalhes precisem ser adicionados a esta dimensão, recomenda-se uma análise financeira mais detalhada, utilizando-se, por exemplo, modelo financeiro baseado em análise de valor presente ou valor futuro. 
Tabela 3. Critérios para avaliação da dimensão N (Negócios).

\begin{tabular}{|l}
\hline CRITÉRIOS PARA AVALIAÇÃO DA DIMENSÃO N (NEGÓCIOS) \\
\hline CRITÉRIOS FINANCEIROS \\
- Custo Inicial com Hardware: Custo inicial com hardware para instalação. \\
- Custo Inicial com Software: Custo inicial com software para instalação. \\
- Custo Inicial com Telecomunicações: Custo inicial com telecomunicações para instalação. \\
- Custo Inicial com Treinamento: Custo inicial com treinamento para operação do produto nas unidades \\
- telecomunicações. \\
Custo mensal com melhorias e modificações: Custo mensal de manutenção e desenvolvimento de novas \\
funcionalidades.
\end{tabular}

A Tabela 4 mostra os critérios que foram inicialmente identificados como mais relevantes na avaliação da dimensão da Organização, resultado da proposta inicial dos pesquisadores, refinada com a experiência dos membros internos da organização adquirente. Existem vários instrumentos que podem prover evidências do comprometimento do fornecedor com a evolução do produto como, por exemplo, a presença de objetivos explícitos de evolução do produto (ou do segmento de negócios do produto) no Planejamento Estratégico da empresa.

Tabela 4. Critérios para avaliação da dimensão O (Organização).

\begin{tabular}{|l}
\hline CRITÉRIOS PARA AVALIAÇÃO DA DIMENSÃO O (ORGANIZAÇÃO) \\
\hline SUPORTE OPERACIONAL \\
Documentação para o usuário final: Existe documentação do produto, organizada de forma a facilitar o acesso pelo \\
usuário. \\
Modalidades de Suporte Operacional: Fornecedor oferece suporte ao usuário através de vários meios: Internet, Call \\
Center, visitas periódicas etc. \\
Agilidade no Atendimento: Fornecedor especifica e garante um tempo médio adequado para a solução de um problema \\
operacional reportado. \\
POLÍTICAS E DIRETRIZES DO FORNECEDOR \\
- Comprometimento com a continuidade do produto: Existem evidências que o fornecedor está comprometido com a \\
- Suntinuidade e evolução do produto. \\
- Puporte Financeiro: Existem evidências que o orçamento do fornecedor será suficiente para a manutenção da operação \\
Priorização de Implementações: Existem evidências de que a evolução do produto será gerida imparcialmente, de \\
forma a garantir que as solicitações concorrentes dos diversos usuários sejam avaliadas, priorizadas e implementadas. \\
Impactos Sociais na Região: Gera conseqüências na região que o fornecedor atua (redução no recolhimento de \\
impostos, redução de empregos, perda de oportunidade de evolução tecnologica na região).
\end{tabular}

\subsection{Definição do instrumento de avaliação}

Uma vez concluída a definição dos critérios a serem utilizados na avaliação, definiu-se o instrumento a ser utilizado. Optou-se por elaborar, para cada um dos atributos, um conjunto de perguntas que permitisse quantificar o atributo e facilitar a investigação das questões de avaliação. Essas perguntas foram organizadas no formato de questionário e a elas foi atribuída uma escala de valores possíveis, variando de 0 (não atende o requisito) até 3 (atende plenamente ao quesito). 
Para a determinação de uma pontuação final foram propostos pesos diferenciados para cada grupo de atributos, uma vez que possuem graus diferenciados de relevância dentro do contexto sendo analisado. A dificuldade em balancear os aspectos a serem considerados de acordo com a situação de avaliação é ressaltada por [FARBEY et al., 1995] e foi plenamente confirmada na prática. Inicialmente, os pesquisadores propuseram uma distribuição de pesos da seguinte forma: 30\% para Negócios, 30\% para arquitetura, 20\% para Processo e $20 \%$ para Organização. Posteriormente, conforme descrito na próxima seção, estes valores não se mostraram aplicáveis completamente ao contexto e foram revistos pela empresa.

Para atribuir um valor (na prática, uma nota) para cada uma das alternativas sob investigação, a proposta foi a utilização da média ponderada entre os diversos atributos das dimensões NAPO, conforme estabelecido na equação da Figura 4.

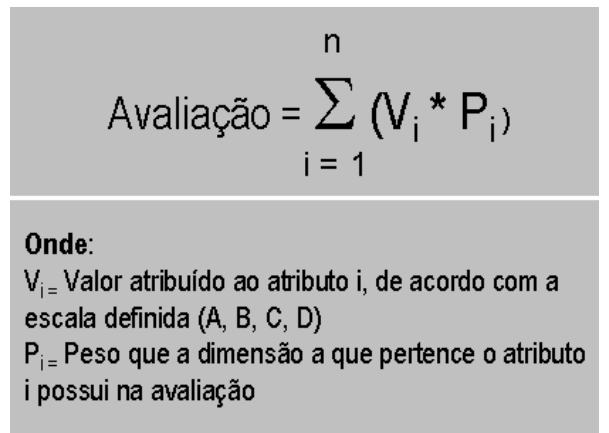

Figura 4. Fórmula para cálculo do valor da avaliação.

\subsection{Análise crítica do modelo em relação ao contexto de aplicação}

Ao observar a escada de benefícios de Farbey apresentada na Figura 2, pode-se, em um primeiro momento, entender que a situação atual da empresa é o degrau 1, ou seja, existem mudanças obrigatórias a serem realizadas em função de uma mudança tecnológica imposta pelo fornecedor. No entanto, ao olharmos mais detidamente as informações do cenário, descritas na seção 3, percebe-se que a situação é um pouco mais complexa porque envolve, não apenas uma análise financeira tradicional, mas sim, as possibilidades que a inserção de uma nova tecnologia poderia viabilizar para a empresa. Uma dessas possibilidades é aumentar o grau de ligação inter-organizacional com os prestadores de serviços (médicos, hospitais etc.) de forma a integrar a cadeia de valor; a outra, a de construir um datawarehouse para auxiliar a tomada de decisão. Portanto, apesar de aparentemente a empresa encontrar-se em uma situação correspondente ao degrau 1, na realidade precisa-se dos elementos de avaliação mais complexos e completos, condizentes com o níveis 5 e 6 . Desta forma, o nível 8 proposto no modelo conceitual encaixa-se adequadamente no contexto.

O uso de atributos combinando as quatro dimensões NAPO pareceu adequado ao contexto de aplicação uma vez que, durante a etapa de obtenção e análise de dados percebeu-se uma necessidade de analisar as alternativas sob um espectro mais amplo do que apenas a avaliação dos aspectos relacionados às funcionalidades do produto em si. Estas preocupações aparecem refletidas nos pesos que foram atribuídos pelos membros internos da organização, descritos na próxima seção. 


\section{Implementação da ação: uso do modelo proposto}

\subsection{Refinamento do cenário e do modelo com base nos especialistas do contexto}

Na prática percebeu-se claramente o ciclo iterativo típico e tão necessário na metodologia da Pesquisa-Ação: foi necessário um novo ciclo de refinamento das alternativas de estratégias de TI e também dos pesos que haviam sido inicialmente definidos para as dimensões do modelo multidimensional.

Esta etapa de refinamento teve iniciou-se com a reavaliação das alternativas de estratégia de TI que seriam pontuadas. Em entrevista com os gerentes da operadora de saúde, duas alternativas iniciais foram imediatamente descartadas. Primeiramente, não deveria ser considerada como uma opção viável a manutenção da situação atual, pois os riscos de se manter uma tecnologia obsoleta e com custos de manutenção elevados não era uma estratégia aceitável. Em segundo lugar, foi retirada a opção de desenvolver sistemas internamente pois esta situação seria mais onerosa, uma vez que se perderia o benefício de utilizar um sistema cooperativo, com custos compartilhados. Restaram, portanto, duas opções de estratégia a serem analisadas: migrar para a nova versão do sistema atual, mantendo o mesmo fornecedor (Estratégia 1) ou migrar para outro fornecedor (Estratégia 2).

Em seguida, devido ao cenário de negócios, constatou-se que o quadro de pesos definidos para as dimensões teria que ser adequado, de modo a refletir a importância de cada dimensão no contexto da aplicação, conforme ilustra a Erro! Fonte de referência não encontrada.. Considerando que, de acordo com as informações prestadas pelos gerentes da operadora de saúde os produtos dos dois fornecedores eram funcionalmente similares, a pontuação da dimensão Arquitetura poderia ser reduzida de $30 \%$ para $15 \%$. Devido a certas dúvidas sobre a capacidade da estrutura de um dos fornecedores, optouse por elevar o peso da dimensão Processo de $20 \%$ para $30 \%$ e a dimensão da Organização passou de $20 \%$ para $40 \%$, pois percebe-se como muito relevante a quantidade e variedade dos meios de suporte operacional. Os critérios de Negócios, aqui basicamente centralizados na questão dos custos, recebeu um peso de $15 \%$.

\subsection{Aplicação da avaliação da estratégia utilizando o modelo multidimensional}

A aplicação do modelo para a avaliação das estratégias inicialmente selecionadas ocorreu em cinco passos:

- Contato inicial com os fornecedores das estratégias para esclarecer os objetivos da pesquisa e os resultados esperados.

- Envio do questionário com as questões para serem preenchidas pelos fornecedores.

- Refinamento das respostas com entrevistas aos fornecedores (uma delas se realizou de forma presencial e a outra por telefone, devido à distância física).

- Pontuação dos quesitos com base na escala definida no modelo (Figura 5), realizada pelos pesquisadores, com base nas respostas ao questionário.

- Tabulação final e discussão dos resultados com a equipe interna da organização.

A Figura 5 apresenta os resultados que foram obtidos com a aplicação do modelo multidimensional, evidenciando a Estratégia1, como sendo a vencedora pelos critérios estabelecidos. 


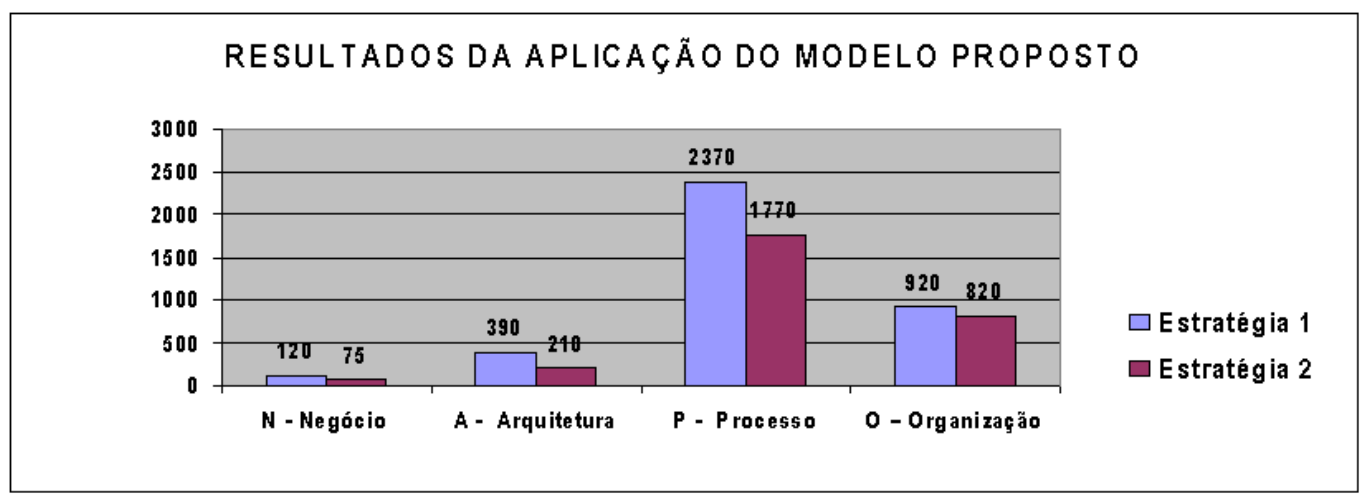

Figura 5. Resultados da aplicação do modelo multidimensional.

\section{Discussão dos resultados}

Tanto o modelo de avaliação utilizado, quanto os resultados obtidos com a avaliação foram analisados criticamente em conjunto pela equipe de pesquisadores e pela equipe interna da organização, conforme recomenda a metodologia da PesquisaAção adotada. Houve um consenso que o modelo multidimensional utilizado refletiu adequadamente o que intuitivamente se esperava como resultado da avaliação de estratégia para este contexto, evidenciando, de forma clara, atributos importantes no processo decisório da empresa.

Iniciando com a análise da dimensão de Negócios, aqui representada pelos custos que cada uma das alternativas traria para a organização, percebe-se que o modelo pontuou adequadamente as duas estratégias. Para a Estratégia 1, como era de se esperar, o custo seria menor, uma vez que não haveria a necessidade de treinar os usuários do produto, pois a maioria dos processos de negócio não sofreria alteração. Também para esta alternativa, ao contrário da segunda, não haveria custo com softwares adicionais para a instalação do novo produto. Os atributos definidos se mostraram adequados para avaliar este aspecto, conferindo vantagem para a alternativa da Estratégia 1.

Com relação à dimensão Arquitetura, o uso de critérios puramente focados nas funcionalidades dos produtos, abordagem bastante comum na avaliação de produtos de software, não teria sido suficiente para que a decisão pudesse ser tomada com segurança. Isto é especialmente relevante neste contexto uma vez que os produtos eram, por premissa, bastante similares em termos de funcionalidade. O uso complementar de atributos ditos não-funcionais (representados no modelo pela avaliação de: confiabilidade, usabilidade, eficiência, manutenibilidade e portabilidade) conseguiu conferir certo grau, embora pequeno, de vantagem para a Estratégia 1.

Conforme se pode observar na Figura 5, o principal critério de desempate acabou sendo a dimensão de Processo, ou seja, a maturidade do processo do fornecedor correspondente à Estratégia 1, em comparação com a maturidade do processo do fornecedor da Estratégia 2. Como esta era uma preocupação grande da empresa adquirente, pois havia indícios de que o processo do fornecedor da Estratégia 2 poderia não suportar o crescimento de usuários esperado, o método mostrou-se adequado para esta dimensão. Este fato veio a comprovar-se posteriormente na prática, quando o fornecedor da Estratégia 2 retirou-se do mercado por não conseguir atender à demanda crescente da base instalada. 
$\mathrm{Na}$ dimensão Organização, também destaca-se a habilidade do fornecedor da Estratégia 1 em lidar com a continuidade do negócio e com o suporte ao uso do produto de forma contínua, o que não foi claramente evidenciado no fornecedor da Estratégia 2. Neste tópico também o modelo adequadamente refletiu a superioridade da Estratégia 1 que era intuitivamente percebida pela equipe da empresa.

Ao analisar as dimensões NAPO selecionadas para compor o modelo de avaliação multidimensional, percebeu-se que os atributos de cada dimensão precisam ser cuidadosamente selecionados, de acordo com o cenário da aplicação. Isto significa que são sensíveis ao contexto e que não podem ser tratados de forma universal e linear. $\mathrm{O}$ uso de pesos para estabelecer uma média ponderada também mostrou-se apropriado pois conferiu grau de precisão maior na identificação de atributos de desempate (especialmente na dimensão de Processo).

\section{Conclusões e trabalhos futuros}

Há alguns anos atrás, as discussões acerca do papel da TI nas organizações foi acirrada pelo polêmico artigo de Carr (2003) afirmando que a TI não agrega mais valor ao negócio, pois tornou-se uma commodity. Embora coerente em suas argumentações, Carr deixa de lado a análise mais profunda no que se refere à flexibilidade proporcionada pela TI e suas aplicações. No caso da empresa analisada, o fato de estar atrelada a uma tecnologia ultrapassada estava limitando suas oportunidades de explorar de forma mais efetiva, a TI. Além disto, manter a situação atual era inviável uma vez que o produto estava sendo descontinuado, passando a gerar prejuízos pela não adequação às novas regulamentações.

Ao analisar a escada de benefícios de Farbey (Figura 2), percebeu-se que, conforme já haviam afirmado os autores, em situações da vida real, as aplicações não se encaixam em apenas um degrau. É necessário que se estabeleçam métodos de avaliação híbridos, multidimensionais, que permitam avaliar a situação da forma mais abrangente possível. Isto foi constatado na aplicação do modelo, uma vez que uma análise que focasse apenas a dimensão da Arquitetura (produto em si) não teria levado a nenhuma conclusão de valor, pois este não seria um critério de desempate relevante, uma vez que os aspectos de funcionalidade eram igualmente cobertos pelos dois sistemas. Foi possível, através dos ciclos de iteração da Pesquisa-Ação, e da aplicação do modelo inicial proposto, refinar aspectos do próprio modelo, como, por exemplo o uso de pesos diferentes dos inicialmente concebidos pelos autores.

Como trabalhos futuros, o objetivo do grupo é analisar outras possibilidades de refinamento da dimensão de Arquitetura, como, por exemplo, a introdução de atributos provenientes do método ATAM, definido em [CLEMENTS et al., 2001]. Posteriormente, utilizar o modelo proposto em outros segmentos de contexto.

Finalmente, pode-se comprovar, na prática, o que já havia sido observado por [BENNET; LEE, 2000] apud [COUGHLAN; COGHLAN, 2002], em um estudo sobre a produtividade na indústria de jornais: "A Pesquisa-Ação não apenas investigou e melhorou a prática gerencial, mas também desenvolveu as competências gerenciais daqueles envolvidos com a pesquisa." 


\section{Referências}

[ABNT, 1996] ABNT - ASSOCIAÇÃO BRASILEIRA DE NORMAS TÉCNCIAS. NBR 13596 - Tecnologia de informação - Avaliação de produto de software Características de qualidade e diretrizes para o seu uso (Versão brasileira da norma ISO/IEC 9126, 1991). Rio de Janeiro: ABNT, 1996, 10 p.

[ANS, 2009] ANS - AGÊNCIA NACIONAL DE SAÚDE SUPLEMENTAR. Dados do Setor, 2009. Disponível em: http://www.ans.gov.br, acesso em 10/01/2009.

[BOSCH, 2000] BOSCH, J. Designing Software Architectures - Adopting and evolving a product-line approach. Harlow: Addison-Wesley Publishing Company, 2000, 354 p.

[BROOKS, 1995] BROOKS, F. The Mythical Man-Month: Essays on Software Engineering - Anniversary Edition. Reading, MA: Addison-Wesley, 1995. 322 p.

[CARR,2003] CARR, N. TI já não importa. Harvard Business Review, pp. 30-37, Maio 2003.

[CLEMENTS et al., 2001] CLEMENTS, P.; KAZMAN, R.; KLEIN, M. Evaluating Software Architectures: Methods and Case Studies. Boston, MA: Addison-Wesley Publishing Company, 2001, 368 p.

[COUGHLAN; COUGHLAN, 2002] COUGHLAN, P.; COUGHLAN, D. Action research for operations management. International Journal of Operations and Production Management, 2002, v.22, n.2. Academic Research Library, 2002, p. 220 240.

[FARBEY et al., 1995] FARBEY, B.; LAND, B.B. \& TARGETT, D. A taxonomy of information systems applications: the benefits' evaluation ladder. 4, 1995, pp. 41-50.

[LAURINDO, 2002] LAURINDO, F.J.B. Tecnologia da Informação - Eficácia nas Organizações. São Paulo: Futura.

[MCFARLAN, 1984] MCFARLAN, W.F. Information Technology changes the way you compete. Harvard Business Review, May-June, 1984, pp. 98-103.

[NOLAN, 1973] NOLAN, R.L. Managing the computer resource: a stage hypothesis. Communications of the ACM 16(7), 1973, pp. 399-405.

[SEI, 2006] SEI - SOFTWARE ENGINEERING INSTITUTE. Capability Maturity Model Integration for Development - Version 1.2 (CMU/SEI-2006-TR-008). Pittsburgh, PA: SEI, Carnegie Mellon University, 2006, 561 p.

[SOFTEX, 2006] SOFTEX - SOCIEDADE PARA A EXCELÊNCIA DO SOFTWARE BRASILEIRO. "MPS.BR - Melhoria de Processo do Software Brasileiro - Guia Geral (v1.1)". Campinas: SOFTEX, 2006, 56p.

[WIJNSTRA, 2002] WIJNSTRA, J. G. Critical Factors for a Successful Platform-Based Product Family Approach. In: SOFTWARE PRODUCT LINES: SECOND INTERNATIONAL CONFERENCE (SPLC), 2., 2002, San Diego, CA. Anais... Berlin, Heidelberg: Springer-Verlag, 2002, p.68-89. (Lecture Notes in Computer Science 2379). 